\title{
Pulmonary resection undertaken with a provisional but unsubstantiated diagnosis of bronchial carcinoma
}

\author{
B. T. LE ROU X \\ Thoracic Unit, Edinburgh
}

\begin{abstract}
Over a 15-year period when frozen-section examination was not regularly available, 918 patients with peripheral pulmonary lesions (the routine preliminary investigation of which did not provide a diagnosis) were managed aggressively by a diagnostic pulmonary resection which, before 1954, was in some patients of the magnitude of pneumonectomy, but after that date was never more extensive than lobectomy and often no more than segmental resection. In 756 patients (82\%) the lesion was shown to be a primary or metastatic pulmonary tumour and in 162 patients (18\%) the lesion was a chronic inflammation or a pulmonary infarct. The commonest pulmonary lesion judged so closely to mimic pulmonary carcinoma that management by exploratory thoracotomy and diagnostic pulmonary resection was mandatory was pulmonary tuberculosis (82 patients) ; other lesions encountered were cavitated ( 8 patients) and non-cavitated (16 patients) pneumonias ; pulmonary gummata ( 2 patients) ; progressive massive fibrosis (4 patients); and a pulmonary infarct ( 5 patients). It is estimated that in perhaps 60 patients $(6.5 \%$ of the total) with nontumorous lesions the pulmonary resection undertaken was needless, or of unnecessary magnitude, and some of these resections might have been avoided if skilled interpretation of frozen sections had constantly been available.
\end{abstract}

Detailed and appropriate investigation of the patient who presents with a single peripheral pulmonary opacity-by clinical and radiographic examination; by comparison of present films with all previous radiographs; by bronchoscopy and perhaps by thoracoscopy; by the bacteriological and histological examination of sputum; and by a period of observation sufficiently long to allow acute inflammatory lesions to clear and yet sufficiently brief to avoid providing a primary pulmonary tumour with the opportunity of growth to an unmanageable size-is commonly unhelpful and there is then the need to have recourse to exploratory thoracotomy, which provides the opportunity for both diagnosis and treatment. How common is it to resect needlessly, because it is not a carcinoma, a peripheral pulmonary lesion?

In the 15-year period 1949 to $1963,4,000$ patients with pulmonary carcinoma were investigated in the regional Thoracic Unit in Edinburgh. Details relating to these patients have been published (le Roux, 1968). In 609 of these patients pulmonary resection-segmental resection in 17 , pneumonectomy in 290 , and lobectomy in 302

1Present address: Thoracic Unit, Wentworth Hospital, Durban was undertaken in the knowledge that the appearances at bronchoscopy were normal, and in these patients it was later established by histological examination of the resected specimen that resection had been undertaken for bronchial carcinoma. In many of these patients the diagnosis of bronchial carcinoma was evident once the chest had been opened. In a further 122 patients, exploratory thoracotomy was undertaken with a view to pulmonary resection in the belief that a peripheral lesion was a carcinoma, and resection was either frustrated because of the extent of mediastinal invasion or rendered pointless by the extent of tumour dissemination. Biopsy of the primary lesion, in all instances where a planned resection was abandoned, showed the presence of carcinoma. Thoracotomy was, therefore, undertaken in 731 patients without preoperative histological confirmation of the diagnosis of bronchial carcinoma other than the finding of tumour cells in the sputum. It became standard practice to search for tumour cells in sputum only after 1955 , but, during the period when sputum was examined in this way, tumour cells were found in the sputa from only $7 \%$ of patients in whom this was the only source of cytological information regarding the nature of a pulmonary lesion before 
operation, and the decision to manage the pulmonary lesion in these patients was made without reference to the finding of tumour cells in the sputum-in fact before cytological reports on the examination of sputa were available, because many reports became available only after thoracotomy had been undertaken and resection completed. Throughout the period under discussion, it was standard practice to manage by exploratory thoracotomy a persistent peripheral pulmonary lesion in a patient suitable in other respects for an operation, in circumstances which made bronchial carcinoma a reasonable, if only an alternative, diagnosis. Failure to find tumour cells in the sputum did not, at any time, alter the decision to manage by exploratory thoracotomy a peripheral pulmonary lesion which, on other grounds, was thought to be a tumour.

As a corollary to this attitude towards the management of peripheral pulmonary lesions judged, on reasonable grounds, by experts possibly to be bronchial carcinoma, it is necessary to record the number of exploratory thoracotomies and pulmonary resections undertaken in the same period and in the same circumstances for lesions either recognized at exploration or shown on histological examination after resection not to be bronchial carcinomata.

Pulmonary resection was undertaken in nine patients for lesions shown on histological examination of the resected specimen to be a sarcoma-unqualified in three, lymphosarcoma in two, fibrosarcoma, either primarily pulmonary with parietal invasion, or vice versa, in three, and rhabdomyosarcoma in one. Distinction between carcinoma and sarcoma, for the purpose of patient management, is probably a quibble.

Isolated peripheral pulmonary metastases from extrathoracic primary tumours were resected in 16 patients, in two sets of circumstances-in patients known to have had a previously managed extrathoracic primary tumour and in patients managed as if the pulmonary tumour was a primary, in whom the diagnosis of pulmonary metastases was made after histological examination of the resected specimens, and in whom the silent primary tumour was demonstrated by appropriate investigation after recovery from pulmonary resection. Of the 16 patients, there was an unsuspected silent primary in four, renal in two, colonic in one, and testicular in one. It was recognized in the remaining 12 patients that the new pulmonary lesion for which investigation was being undertaken might represent a metastasis from a primary extrathoracic tumour managed 6 to 17 years earlier. Other metastases were not demonstrated by clinical or special investigation in this group of patients, and the pulmonary lesion was, therefore, resected-by lobectomy in nine patients and by pneumonectomy in three. Metastases-from renal carcinoma in four, from mammary carcinoma in five, from carcinoma of the bladder in two, and from carcinoma of the uterus in one-as the cause of the pulmonary lesion were demonstrated on histological examination of the resected specimens.

In the same period a carcinoma, unequivocally primary in the lung, was resected in three patients previously managed surgically for mammary carcinoma, in four patients previously irradiated for carcinoma of the larynx, in two patients who had had a colectomy for carcinoma, in three patients managed by oesophagogastrectomy for carcinoma of the gastro-oesophageal junction, in 17 patients previously managed by irradiation or excision for malignant skin tumours, in two patients who had undergone hysterectomy for carcinoma, in a woman who had been irradiated for a skin tumour, managed by mastectomy for mammary carcinoma, and later died from a gastric tumour, and in a man whose peripheral malignant melanoma had been managed by amputation of the leg.

A pulmonary lesion which develops in a patient previously managed for a malignant extrathoracic tumour is likely, but not certain, to be a pulmonary metastasis. If the pulmonary lesion is not a metastasis, to neglect the opportunity of managing it surgically at an early stage is unfortunate-and in these circumstances lesions are often detected at an early stage because patients in whom a tumour has been dealt with surgically or by irradiation are usually closely followed. If a pulmonary lesion proves to be a metastasis, its resection should not seriously modify the prognosis by shortening life if it is one of many metastases, the others not having been recognized; and if it is the only metastasis, long survival may be achieved. Long survival following resection of a metastasis, and possibly even of the primary tumour, is more likely to reflect the natural behaviour of a particular tumour than surgical proficiency. Some patients harbour slowly growing malignant tumours for decades.

Lobectomy for metastasis from mammary carcinoma was undertaken in three women, 6, 9, and 14 years respectively after mastectomy for the primary tumour; in these three patients other metastases became evident 4,8 , and 11 years respectively after resection of the pulmonary metastasis. In two of the three patients the exhibition of appropriate hormone therapy was followed 
by clinical and radiographic restoration to normal; the third patient died within three months with widespread metastases.

In the same period peripheral pulmonary lesions were recognized, without recourse to exploratory thoracotomy, as metastatic in 104 patients who had been managed earlier for a primary extrathoracic tumour. Recognition of the pulmonary lesion as metastatic was possible because of related lymph node metastases; multiplicity of the pulmonary lesion; related pleural effusion which allowed of the replacement of the effusion with air, thoracoscopy, and biopsy; by biopsy of parietal pleura during management of empyema thoracis by open drainage; or by biopsy of an associated rib lesion. The commonest site of the original primary tumour was the breast (42 patients), kidney (12 patients), colon (9 patients), cervix or body of uterus (8 patients each), and prostate and rectum (5 patients each); there were one or two examples of pulmonary metastases from previously managed tumours in several other sites. In only three patients did pulmonary metastases from an extrathoracic primary erode a bronchus and become visible at bronchoscopy-that is, present as a central type of tumour from which an informative biopsy could be made at bronchoscopy. While bronchial compression, particularly with intrathoracic metastases from mammary carcinoma, is not uncommon, the bronchial mucosa is, in these circumstances, usually normal and information of value is rarely obtained from a biopsy made at bronchoscopy.

Also, in the same period, 49 other patients with pulmonary metastases were investigated in whom the site of the primary tumour was not found until necropsy, which in 32 demonstrated a small unsuspected primary-in gut, thyroid, pancreas or suprarenal. In this group of 49 patients the lung was only one of many sites of tumour spread, and in all but 11, histological evidence of a malignant tumour was found on examination of biopsy material obtained by lymph node resection, pleural biopsy at thoracoscopy, or other manoeuvre, but the histological appearances were not sufficiently well differentiated to ascribe the primary tumour to a particular site.

A variety of chronic inflammatory pulmonary lesions may mimic or co-exist with pulmonary carcinoma. Thus, pulmonary carcinoma and active pulmonary tuberculosis are known to have coexisted in 69 patients-one a boy of 19 yearsbut in some of these patients the tumour was not a surgical problem. Exploratory thoracotomy was undertaken, in the period under discussion, in 82 patients in all of whom sputum examination be- fore operation had failed to demonstrate the presence of either tumour cells or tubercle bacilli. In all, the pulmonary lesion was peripheral in type and the appearances at bronchoscopy were normal. Radiography had failed to show evidence such as calcification or satellite shadows which made more likely the diagnosis of pulmonary tuberculosis. In many, the diagnosis of carcinoma was favoured because the pulmonary lesion was cavitated and antituberculosis drugs had not yet been exhibited-a combination of circumstances which, had the lesion been tuberculous, made it reasonable to expect a positive sputum. At exploratory thoracotomy in these 82 patients, 62 of whom were men, the lesion was thought most likely to be tuberculous in 45 who were, therefore, submitted only to segmental resection. Lobectomy was undertaken in 26 and pneumonectomy in 8 patients, still with the diagnosis of bronchial carcinoma foremost in the mind of the surgeon; all were shown to have pulmonary tuberculosis on histological examination of the resected specimen. In three patients the lesion was found to be an interlobar empyema and this was enucleated. Three of the 82 tuberculous lesions managed in the circumstances outlined were resected in 1949, and the number of resections in each subsequent year including 1963 were $4,3,4,6,4,9,9,9,7,6$, $6,5,2,5$. In 39 of the 82 patients there were available, for comparison with recent radiographs, films taken two to six years before presentation to the Thoracic Unit, and one of the reasons for favouring the diagnosis of carcinoma in these patients was the normality of the earlier radiographs. Segmental resection is not an excessive resection for pulmonary tuberculosis in circumstances which demand exploratory thoracotomy-especially as it has always been our practice to undertake this variety of exploratory operation with the patient under treatment with the three standard antituberculosis drugs - chemotherapy beginning some days before operation and continuing at least until the histological report on the resected specimen is available. In the same circumstances, lobectomy is also probably not an excessive resection. If the diagnosis of tuberculosis had been established, segmental resection, rather than lobectomy, might have been undertaken in some of these patients. Pneumonectomy, on the other hand, is an acceptable operation for pulmonary tuberculosis only if the lung is unequivocally destroyed. The eight pneumonectomies were all undertaken at a time when a resection of this extent was virtually standard for pulmonary carcinoma-before 1953 -and they were undertaken because the surgeon believed that bronchial carcinoma was the cause of 
the lesion found at thoracotomy. It is most unlikely that pneumonectomy would ever again be undertaken for a tuberculous lesion, in the circumstances under discussion, in the absence of surgical accident or error during a smaller resection.

Progressive massive fibrosis was commonly recognized in the period under discussion on chest radiographs of miners referred for other purposes; 67 patients were referred specifically for the investigation of a pulmonary lesion thought by referring physicians unlikely to be progressive massive fibrosis and most likely to be a pulmonary carcinoma. In 63 of these patients, either additional information was gathered in the Thoracic Unit which made the diagnosis of progressive massive fibrosis reasonably certain and obviated the need for exploratory thoracotomy, or other circumstances precluded exploratory thoracotomy and the subsequent progress of the lesion established the diagnosis of progressive massive fibrosis. In four patients the recent appearance and the rate of growth of a unilateral pulmonary lesion made the diagnosis of pulmonary carcinoma so likely that lobectomy was undertaken and all survived resection; in all, histological examination of the resected specimen established the diagnosis of progressive massive fibrosis; clearly, none could afford unnecessarily to lose functioning lung.

In the period under discussion the diagnosis of pulmonary infarction was made in 127 patients referred to the Thoracic Unit for the investigation of haemoptysis, pleural effusion, a pulmonary opacity or a combination of these abnormalities. In five other patients, explora ${ }^{+}$ory thoracotomy was undertaken in the belief that the cause of a pulmonary opacity was a peripheral carcinoma; the findings in one were sufficiently suggestive of carcinoma for lobectomy to be undertaken; in four, the lesion was thought on exploration so unlikely to be a carcinoma that a segmental resection was made; one of these last-mentioned four died from coronary thrombosis on the fourth postoperative day. The histological diagnosis from the resected specimens in these five patients was pulmonary infarction.

Two female patients in whom a large peripheral pulmonary lesion was managed by pneumonectomy-one in 1949 and one in 1951-were shown to have had a pulmonary gumma on histological examination of the resected specimens. In neither had appropriate serological examinations been made during investigations to establish the diagnosis of syphilis. One of these patients died at operation from haemorrhage, the consequence of damage to the azygos vein.
Of 81 patients managed for acute or chronic lung abscess in the period under discussion, 17 required pulmonary resection; of these 17 patients, eight fall into the category of patients submitted to thoracotomy and pulmonary resection because a radiographically cavitated peripheral pulmonary lesion was thought most likely to be a pulmonary carcinoma. All were relatively well; in none was there a history suggestive of acute lung abscess; all are probably better classified as having chronic destructive pneumonia. Lobectomy was undertaken in six of these patients and pneumonectomy in two. Of the eight patients, one died from brain abscess and one from meningitis after a complicated convalescence; one died after a series of events-empyema as a complication of the original lobectomy, bronchopleural fistula, completion of pneumonectomy as the only way of managing the fistula, and finally death from secondary haemorrhage from the pulmonary artery. Postoperative empyema complicated recovery in three of the remaining five.

A large number of patients $(1,083)$ was referred to the Thoracic Unit in the period under review because a non-cavitated pulmonary lesion failed to show radiographic evidence of clearing soon enough to make acceptable the diagnosis of 'pneumonia'. Subsequent behaviour in most of these patients made reasonably certain the diagnosis of a non-specific pulmonary infection and complete clearing of the opacity made unlikely the diagnosis of pulmonary carcinoma; all had normal bronchi. Inevitable delay in referral after clinical suspicion had initially been raised rarely made it necessary to reach a decision on management in a patient with a peripheral pulmonary shadow without a series, for comparison, of at least three chest radiographs made over five to seven weeks. Progressive and unequivocal diminution in size and density of a pulmonary shadow diminishes the likelihood of the shadow being the consequence of a pulmonary carcinoma but does not render this diagnosis unacceptable. Continued surveillance is necessary. In 61 of the 1,083 patients, a pulmonary shadow failed to diminish or increase in size; collateral evidence, such as finger clubbing, haemoptysis, loss of weight, and so on supported the diagnosis of bronchial carcinoma; in this group of patients diagnostic thoracotomy was undertaken together with pneumonectomy in 12 (all before 1954), lobectomy in 29, segmental resection in 12 , and biopsy only in 8 . In all, histological examination of the resected specimen showed the lesion to be a non-specific chronic inflammation. Of the 61 patients, 55 were male smokers. Two men died as the direct consequence 
of operation-pneumonectomy in one and lobectomy in the other-each from respiratory infection and respiratory insufficiency. Two patients in this group who had been submitted to lobectomy later developed pulmonary carcinoma-one in the same lung from which the lobe had been resected and the other in the opposite lung -8 and 10 years after the original operation, respectively. In these two patients the possibility must be recognized that the original lesion for which resection had been undertaken was in fact a carcinoma, and that histological material submitted had been misinterpreted, inadequately scrutinized, or incomplete; the original specimens are no longer available for scrutiny and the slides which remain do not show evidence of tumour; these rare instances justify the preservation of all resected specimens.

From these figures some facts can be extracted. In the period under review, exploratory thoracotomy was undertaken in 918 patients because each had been found to have a persistent peripheral pulmonary shadow which, in the opinion of experts, might have been caused by a pulmonary tumour. Of these 918 patients, $756(82 \%)$ were shown to have a pulmonary tumour-bronchial carcinoma in 731, sarcoma in 9, and an isolated pulmonary metastasis from an extrathoracic primary tumour in 16 . The remainder-162 patients $(18 \%)$-were shown to have some form of chronic inflammatory lesion or a pulmonary infarct. In this group of 162 patients management at exploratory thoracotomy was by segmental resection, pulmonary biopsy, or the enucleation of an interlobar empyema in 72 , because the macroscopic features of the lesion made unlikely the diagnosis of pulmonary carcinoma. Lobectomy was undertaken in 66 patients either because the lesion was still thought most likely to be a tumour or because its size precluded a smaller resection. Pneumonectomy was undertaken in 24 patients in the belief that the lesion was a pulmonary tumour. Seven of the 162 patients died in the postoperative period, two after pneumonectomy, four after lobectomy, and one after segmental resection. Half of the patients who were shown not to have a pulmonary tumour were shown to have pulmonary tuberculosis.

In this series, diagnostic thoracotomy was, in most patients, synonymous with diagnostic pulmonary resection. Diagnostic pneumonectomy was not undertaken after 1954. Examination of frozen sections was not a part of the routine but was occasionally made available by the accessibility of appropriate pathologists. It is difficult to define the place of frozen-section examination in the management of peripheral pulmonary lesions. If a skilled service is routinely and consistently available, it will obviously be used and some needless resections will be avoided. In this series diagnostic pneumonectomy would probably always have been avoided; a needless resection of this extent has not been undertaken since 1954-probably for no other reason than the change in attitude towards the extent of resection by which pulmonary carcinoma is adequately managed, lobectomy now being, where possible, preferable to pneumonectomy. Where the diagnosis of pulmonary tuberculosis is made by examination of frozen sections, there can be few surgeons who would not complete the resection of an isolated lesion, the diagnosis of tuberculosis having been established during operation and exploration having been undertaken in a patient receiving adequate antituberculosis chemotherapy. All the segmental resections and some of the lobectomies for tuberculosis in this series would, therefore, have been made despite the availability of an histologist skilled in the interpretation of frozen sections. Some of the lobectomies for tuberculosis would probably have been less extensive resections. It is estimated that 60 of the 90 resections greater than segmental resection would have been avoided or would have been smaller resections if an accurate histological diagnosis had been supplied once the thoracotomy had been made. Therefore, of the 918 resections undertaken in patients with peripheral pulmonary shadows, $60(6.5 \%)$ may be regarded as unneces. sary resections or resections of an unnecessary magnitude. It is calculated that some of these resections might have been avoided if skilled interpretation of frozen sections had constantly been available. It must, however, always be recognized that a biopsy made at thoracotomy from a large lesion for the purpose of examination of a frozen section may not be representative of the whole lesion, and that, in many instances, biopsy for the purpose of frozen section may well amount to a formal segmental resection or lobectomy. The point which must be clearly established is that diagnostic pneumonectomy is never likely to be a justifiable procedure and, in this series, diagnostic pneumonectomy was not undertaken after 1954.

\section{REFERENCE}

le Roux, B. T. (1968). Bronchial carcinoma. Thorax, 23, 136. 